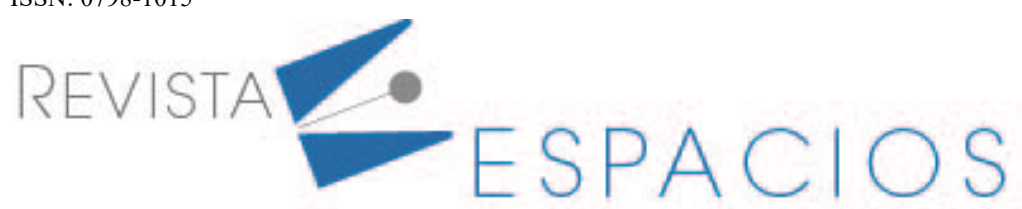

\title{
Análisis acerca de la resolución de problemas matemáticos en contexto: estado del arte y reflexiones prospectivas
}

\section{Analysis about the definition of mathematical problems in context: state of the art and prospective reflections}

DÍAZ, Luis M. ${ }^{1}$

CAREAGA, Marcelo P. ${ }^{2}$

\begin{abstract}
Resumen
Debido a la relevancia de la resolución de problemas en educación matemática, junto con el creciente interés por los estudios comparativos internacionales y su influencia en los sistemas educativos y, por consiguiente, en las currículas escolares, el presente artículo describe los diversos conceptos y significados que se le han atribuido a la resolución de problemas matemáticos en contexto y a su papel en el ámbito de la educación, proponiendo un modelo teórico respecto de cómo reenfocarlos desde una racionalidad bio-ecológica.
\end{abstract}

Palabras clave: problema, matemática, contexto, educación.

\begin{abstract}
Due to the relevance of problem solving in mathematics education, together with the growing interest in international comparative studies and its influence on education systems and, consequently, on school curricula, this article describes the various concepts and meanings that They have been attributed to solving mathematical problems in context and to their role in the field of education, proposing a theoretical model regarding how to refocus them from a bio-ecological rationality.

Keywords: problem, mathematics, context, education
\end{abstract}

\section{Introducción}

En la última década se ha construido una base crítica respecto de los procesos de enseñanza que considera el conocimiento matemático formal, el enseñado por el docente, y la aplicación en la solución de diferentes tipos de problemas aritméticos, en donde el alumno debe desarrollar la habilidad para enfrentar una situación nueva y proponer una solución utilizando los contenidos vistos en el aula (Lerman, 2001; Díaz y Poblete 2001; Jaworski, 2006; Darragh, 2016).

Las investigaciones de Ramos y Font (2006), muestran la existencia de una brecha entre las matemáticas que son explicadas por el docente y la utilización que los alumnos realizan en la vida cotidiana. Los autores D’Amore y Pinilla (2001), plantean que la existencia de esta brecha, teórica y práctica, es uno de los motivos que explican los bajos resultados que los alumnos obtienen en las matemáticas.

La falta de aplicabilidad de las matemáticas en la vida habitual, ha estimulado a un grupo de investigadores a fomentar el uso y aplicación de esta área del conocimiento en situaciones cotidianas (Boaler, 1993), como una

\footnotetext{
${ }^{1}$ Universidad Católica de del Maule (UCM), Chile. Doctorado en Educación, Idiaz.educa21@gmail.com

2 Facultad de Educación y CIEDE. Universidad Católica de la Santísima Concepción (UCSC), Chile, mcareaga@ucsc.cl
} 
educación matemática orientada a la práctica, siendo el objetivo principal desarrollar la capacidad de los estudiantes para aplicar esta área en la vida cotidiana (Graumann, 2011), que se considera un objetivo central de la educación matemática (Biembengut, 2007; Greer et al., 2007), en donde, para que sea efectivo el aprendizaje en la enseñanza de la matemática, se debe apelar a contextos o ejemplos cercanos a las experiencias de los alumnos; permitiendo comprender las reales implicancias que el conocimiento matemático tiene en la realidad (Aravena y Caamaño, 2007; Stein et al., 2008).

La resolución de problemas matemáticos, necesita nuevas visiones paradigmáticas para orientar sus métodos y sus formas de enseñar y de aprender. Se requiere superar la tendencia a la abstracción pura, buscando su aplicabilidad en la vida y en los contextos reales en los que se vive. Se necesitan nuevas taxonomías orientadoras, basadas en sólidos argumentos teóricos y reconceptualizaciones, que permitan explorar una teoría matemática que facilite despejar su cientificidad en el arraigo contextual de sus problemas, para no quedar reducida a la sola formulación de enunciados abstractos.

\section{Metodología}

El presente estudio, se llevó a cabo con una clasificación basada en un análisis de contenido según un objetivo de tipo descriptivo, el cual pretende responder a la interrogante, ¿Qué se entiende por aplicación de problemas matemáticos en contexto?, en base a los criterios propuestos por Piñuel (2002), que implican la selección de la comunicación que será estudiada; la selección de las categorías que se utilizarán; la selección de las unidades de análisis y la selección del sistema de recuento o de medida.

El parámetro de medición es cualitativo transversal, analizando la presencia o ausencia de categorías, su unidad temática es conceptos y referencias en concordancia con el registro y análisis, lo que permitirá en definitiva dilucidar la interrogante planteada (Díaz, 2018).

Figura 1

Diseño de análisis de

contenido cualitativo

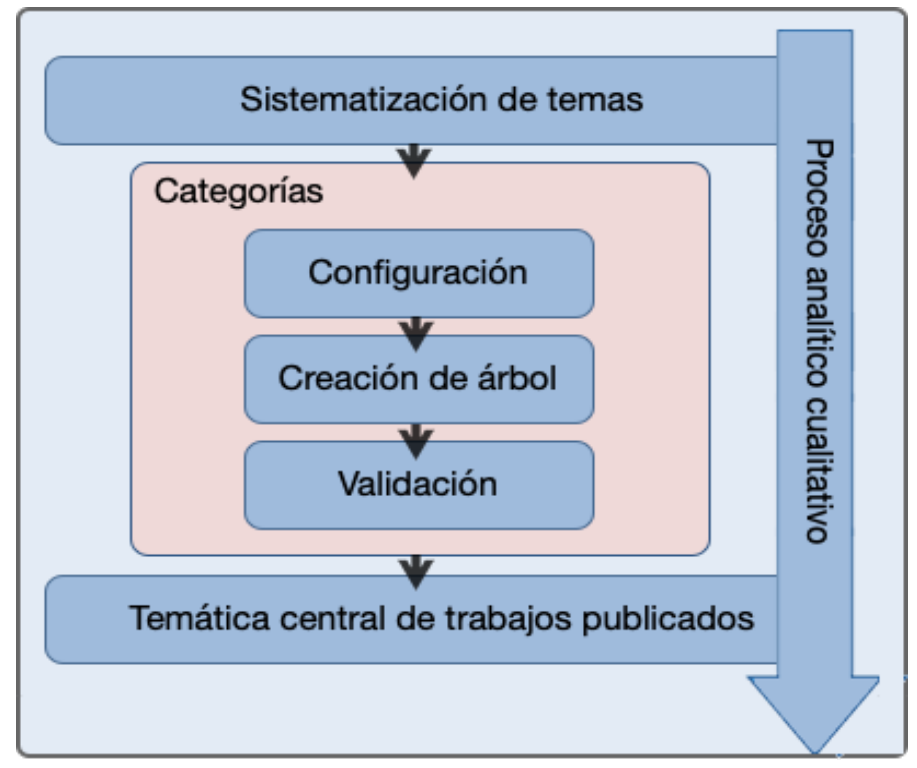

Fuente: Díaz (2018). http://dx.doi.org/10.5209/RGID.60813

En relación al diseño de análisis, éste fue elaborado mediante un proceso analítico cualitativo, el cual se llevó a cabo como una primera etapa, que consideró un procedimiento metodológico sistemático de temas, seguido de 
la caracterización de configuración, creación de árbol y validación, concluyendo con la temática central de trabajos publicados.

\section{Categorías de análisis y resultados}

El análisis de contenidos de los documentos se desarrolló considerando categorías de análisis organizadas a partir de una interrogante y tres categorías generales:

¿Qué se entiende por aplicación de problemas matemáticos en contexto?

1: Matemáticas en contexto.

2: Problemas matemáticos en contexto.

3: Perspectivas de futuro.

\section{1. ¿Qué se entiende por aplicación de problemas matemáticos en contexto?}

En el área de la educación, cada disciplina aborda el estudio de aplicación y resolución de problemas matemáticos con una visión propia, desarrollada por filósofos, psicólogos, matemáticos, especialistas en educación y didáctica (Piaget 1969, Vygotski 1934, Kurt 1931, Russell 1903). Esta situación provoca que, en la actualidad, nos encontremos, con una considerable cantidad de investigaciones centradas en resolución de problemas, cuya completa sistematización, está aún por concluir (Silver 1985; Castro 2008).

Las investigaciones de Ramos y Font (2006), muestran la existencia de una brecha entre las matemáticas que son explicadas por el docente y la utilización que los alumnos realizan en la vida cotidiana. La existencia de esta brecha, es uno de los motivos que explican las actitudes negativas o ansiedad que muchas personas desarrollan hacia las matemáticas (Díez 2004; D’Amore y Pinilla, 2001).

En un estudio realizado, por Crespo (2003), se analizó el razonamiento matemático de 277 estudiantes de 18 años, donde sólo 13 alumnos (4,7\%) dieron respuesta correcta a todos los ítems. Esta situación deja en evidencia la dificultad que poseen los alumnos al finalizar la enseñanza secundaria, y cómo ésta afecta a los alumnos que, pese a tener buenos resultados académicos, presentan debilidad en el razonamiento matemático, generando creencias erróneas sobre la forma de encarar la resolución de ejercicios matemáticos.

En opinión de Díaz (2003) y Jones et al., (2007), sólo una minoría de estudiantes universitarios analiza los problemas matemáticos desde el punto de vista real, utilizando correctamente los procedimientos necesarios para el cálculo de un suceso planteado en un problema.

En la investigación realizada por Leal y Bong (2015), a estudiantes de enseñanza universitaria que habían cursado el ramo de matemáticas fundamental, donde los estudiantes recibieron un problema, que implicó el diseño de un puente, los alumnos no fueron capaces de vincular los conocimientos que poseían, para resolver este problema, pero sí para responder preguntas cerradas. Los resultados demostraron que eran incapaces de visualizar un puente como un problema, por tanto, no pudieron formular hipótesis adecuadas o crear una resolución acertada, lo cual demuestra que la contextualización de los problemas matemáticos continúa siendo un desafío por resolver.

Un caso similar sucedió con la investigación de Lithner (2004), donde examinó el razonamiento matemático de estudiantes universitarios de primer año, en un escenario similar a los exámenes presentó problemas reales, los resultados obtenidos expresaron que los estudiantes no desarrollan estrategias nuevas, relacionadas con los conceptos implicados en el problema propuesto, más bien utilizaron un razonamiento superficial basado en experiencias previas y que los llevó a repetir algoritmos enseñados o utilizados, lo cual marca una tendencia hacia la estandarización en la resolución de problemas matemáticos. 
La tendencia generalizada a no aplicar necesariamente las matemáticas en la vida cotidiana y a sus contextos que la rodean, ha estimulado a un grupo de investigadores a fomentar el uso de esta área en situaciones cotidianas (Boaler, 1993), como una educación matemática orientada a la práctica, siendo el objetivo principal de este movimiento desarrollar la capacidad de los estudiantes para aplicar esta área en la vida cotidiana (Graumann, 2011), que se considera un objetivo central de la educación matemática (Biembengut, 2007; Greer et al., 2007), en donde, para que sea efectivo el aprendizaje en la enseñanza de la matemática, se debe apelar a contextos o ejemplos cercanos a las experiencias de los alumnos; permitiendo comprender las reales implicancias del conocimiento matemático en los contextos en que se vive (Aravena y Caamaño, 2007; Stein et al., 2008).

A través de los enunciados mencionados, se puede obtener una definición de resolución de problemas, pero se debe tener en consideración que son definiciones referidas a problemas genéricos, ya que estos estudios están centrados en los problemas o situaciones de tipo matemático utilizados en el aula.

Castaño (2014), pone de manifiesto la necesidad de buscar alternativas diferentes a las tradicionales utilizadas en el aula, en opinión del autor, se requiere una búsqueda de estrategias, en la que emerjan categorías, como la utilización de la realidad y la perspectiva del estudiante de acuerdo con los modelos de procesamiento de información, con énfasis en las ventajas del aprendizaje contextualizado que involucra la construcción del conocimiento, con los saberes previos del estudiante y la relación con el entorno. Al respecto, Fuenlabrada (2005) enfatiza que los alumnos deben ejercitar a través de formas novedosas y particulares, resolviendo problemas más allá de los métodos tradicionales, con la finalidad que los alumnos logren acercamientos creativos socializando sus procedimientos con sus compañeros en el desarrollo de estrategias, explotando la zona de desarrollo próximo sugerida por Vygotsky (1934). Para (Ortiz, 2005), la definición adecuada de un problema matemático depende, de la disponibilidad de una amplia gama de estrategias que podemos aplicar en diversos contextos y la capacidad de reconocer el problema que debemos resolver. Según Arcavi y Friedlander (2007), la resolución de problemas y la influencia en los sistemas de educación matemática es diferente, varía según la cultura, sistema de educación, currículum, profesores, investigadores, en la enseñanza aprendizaje de las matemáticas y los matemáticos que no necesariamente comparten los mismos puntos de vista, de cómo un problema matemático en contexto debe ser aplicado en el aula. A más de tres décadas de presencia de la resolución de problemas en la agenda de investigación y reformas curriculares, resulta paradójico que su definición e identidad, siga siendo un tema de discusión en la educación matemática (Santos, 2007).

\subsection{Problemas matemáticos}

La importancia de la resolución de problemas, es reconocida internacionalmente como un aspecto central del proceso de aprendizaje en matemáticas y sigue siendo la principal preocupación de educadores e investigadores en Educación (Díaz y Poblete, 2001; Liljedahl et al., 2016).

Lo expuesto, ha influido notablemente en las agendas de investigación en educación matemática, en propuestas del currículum y las prácticas de instrucción (Santos, 2008). Esta situación es producto de la importancia que tiene en el desarrollo de competencias para la vida, reflejado en diferentes documentos nacionales e internacionales que resaltan su valor y la necesidad del desarrollo de esta competencia (Informe Cockcroft, 1985; PISA, 2006; MINEDUC, 2019).

Por tal motivo, es importante aclarar que se entiende por resolución de problema matemático, existen diferentes concepciones definidas sobre lo que es un problema desde la educación, autores como Pozo y Monereo (1999) y Schoenfeld (1988), presentan lo complejo de la conceptualización del término, planteando que un problema, es una situación que precisa una solución, pero que no tiene un camino a un resultado rápido y directo, en donde se deben tomar decisiones que permitan aproximarse cada vez más a la solución requerida, 
considerando estrategias heurísticas, naturaleza del pensamiento matemático, creencias de estudiantes y la relevancia de las estrategias metacognitivas. Para Orton (1998), la resolución de problema es la generación de un proceso mental, en el cual el aprendiz combina variedad de elementos, conocimientos, destrezas, habilidades, capacidades, reglas y conceptos adquiridos de manera previa que admiten dar solución a una situación nueva. Sin embargo, Delgado (1999), afirma que el resolver problemas es una habilidad matemática, que permite encontrar un método o vía que conduzca a la solución del problema.

En opinión de Callejo (1998), en la situación problema se debe buscar, investigar, establecer relaciones e implicar afectos que posibiliten el planteamiento de estrategias de solución al problema. Por tanto, el concepto de problema es relativo al sujeto y al contexto al que se plantea. En esta línea, otros autores se han enfocado hacia aspectos relacionados con la relevancia de considerar situaciones auténticas o realistas, que propicien en los estudiantes la construcción de modelos matemáticos reales (Gravemeijer y Doorman, 1999).

La resolución de problemas, debe ser comprendida como el proceso de interpretar una situación matemática cercana, involucrando ciclos interactivos de expresar, probar y revisar interpretaciones del mundo que lo rodea, revisando o redefiniendo grupos de conceptos matemáticos asociados a la realidad desde varios tópicos, considerando un proceso de reflexión donde el estudiante en forma constante transforme sus ideas y formas de pensar, desarrollando recursos, estrategias, y herramientas que le permitan recuperarse de dificultades reales, con la finalidad de fortalecer sus formas de pensar acerca de su propio aprendizaje (Lesh y Zawojewski, 2007; Santos, 2008).

\subsection{Problemas matemáticos en contexto}

La importancia que tiene contextualizar el conocimiento es ampliamente asumida, ya que se considera que el contexto puede ser la clave para relacionar lo que los psicólogos han aprendido sobre el modo en que los humanos razonan, sienten, recuerdan, imaginan y deciden con lo que, por su parte, han aprendido los antropólogos sobre la manera en que el significado es construido, aprendido, activado y transformado (Font, 2007; Mthethwa, 2019).

Los contextos son aportes importantes para el aprendizaje de las matemáticas, ya que ofrecen diferentes oportunidades en el proceso de adquisición de conocimientos de los estudiantes. El uso de contextos reduce la percepción, que los aprendices tienen de las matemáticas, como un cuerpo remoto de conocimiento (Boaler, 1993), a través de los contextos, los estudiantes pueden desarrollar una mejor comprensión de la utilidad de las matemáticas para resolver problemas de la vida diaria (De Lange, 1987). Para Van den Heuvel-Panhuizen (2001), el contexto social de los estudiantes en el proceso de enseñanza y aprendizaje, las experiencias diarias y las estrategias informales también pueden usarse para introducir conceptos matemáticos. Al resolver un problema basado en un contexto conocido, el alumno puede recrear una situación problemática a través de sus experiencias previas, utilizando estrategias de resolución formales o informales, con la finalidad de encontrar un resultado, dando respuesta a un problema cercano a su realidad, aproximando la matemática a las situaciones cotidianas (De Lange, 1987; Gravemeijer y Doorman, 1999; Van den Heuvel-Panhuizen, 2001). Según lo expresado por Van den Heuvel-Panhuizen y Bodin-Baarends (2004), la educación matemática, puede implicar diferentes tipos de contextos reales, situaciones de fantasía o incluso situaciones del mundo formal de las matemáticas, estas situaciones no están restringidas a entornos del mundo real. Un contexto debe proporcionar información que pueda organizarse matemáticamente, y ofrecer oportunidades para que los estudiantes, trabajen dentro de un ambiente conocido utilizando sus conocimientos y experiencias preexistentes. Para Maass, (2010), el proceso comienza y termina con una solución del mundo real, considerando siete pasos: i) un solucionador necesita establecer un modelo de situación para comprender el problema del mundo real; ii) el modelo de situación se desarrolla luego en un modelo real a través del proceso de simplificación y estructuración; iii) el solucionador necesita construir un modelo matemático al matematizar el modelo real; iv) establecer el 
modelo matemático; v) el solucionador puede llevar a cabo procedimientos matemáticos para obtener una solución matemática del problema; vi) la solución matemática tiene que ser interpretada y validada en términos de problemas reales; $y$, finalmente, la solución del mundo real debe presentarse en términos de la situación del problema en el mundo real.

En el Programa para la Evaluación Internacional de Estudiantes (PISA), los problemas matemáticos son situaciones conflictivas cuya solución no resulta evidente, las estrategias de resolución de problemas son muy variadas y dependen, del contexto donde se desarrolla el alumno. Conscientes de esta situación PISA, plantea distintos tipos de contextos cotidianos e infrecuentes, con la idea que los alumnos, se enfrenten con diversas situaciones problemáticas, y activen los procesos cognitivos más eficaces, para la situación concreta que tienen que resolver.

Desde el año 2012 PISA incorporó la evaluación de las matemáticas basada en computadora (CBAM), el nuevo conjunto de ítems reflejó el uso y la aplicación de las matemáticas dentro de contextos auténticos, utilizando la tecnología para hacer diferentes tipos de preguntas, evaluó diferentes aspectos de la alfabetización matemática, que realmente no es posible hacer con una evaluación en papel, con algunas preguntas específicamente dirigidas al pensamiento de orden superior y las habilidades de resolución de problemas dentro de un entorno informático, incorporó cálculos, que podrían automatizarse 'detrás de escena'; habilidades espaciales y visuales evaluadas usando simulaciones y manipulativos que no están disponibles en formatos tradicionales de papel; estrategias de resolución de problemas (OCDE, 2014; Hoogland, 2018).

PISA, ha definido la competencia resolución de problemas, como la capacidad del individuo, para emprender procesos cognitivos, con el fin de comprender y resolver situaciones problemáticas en las que la estrategia de solución no resulta obvia. Existen tres aspectos clave para elaborar las actividades de evaluación: i) el contexto; ii) la naturaleza; y iii) los procesos de resolución del problema (ver Figura 2). Los diferentes contextos del problema, se refieren a que aquel sea tecnológico o no, personal o social. Lo que determina la naturaleza del problema, es si la información sobre dicha situación, se da a conocer a quien resuelve el problema es completa (problemas estáticos), o si la interacción con esa situación es una parte necesaria de la actividad de resolución para descubrir información adicional (problemas interactivos). (OCDE, 2014).

Figura 2

Características del marco

de resolución de problemas

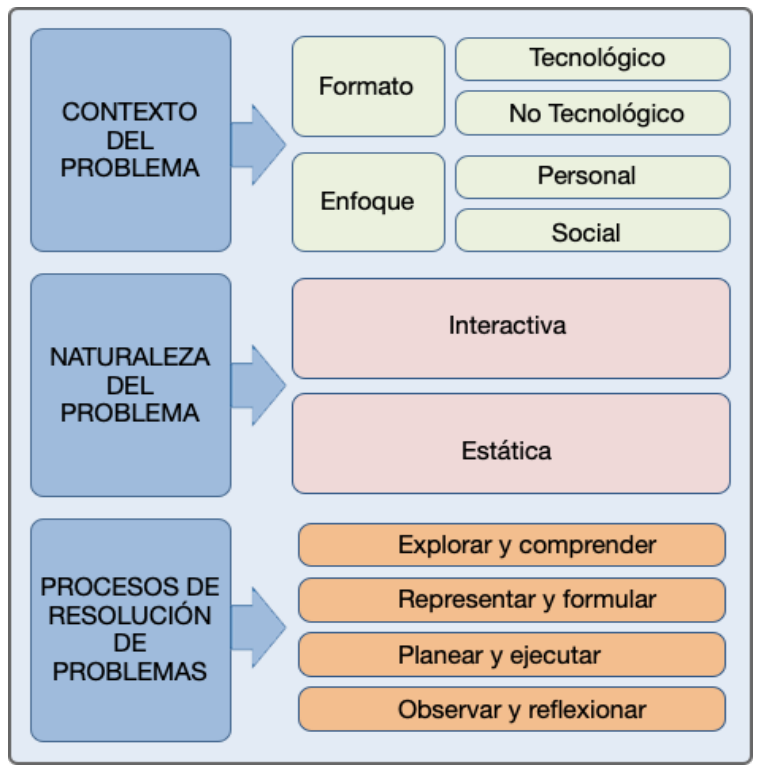

Fuente: PISA (2012) 
En este ámbito la Organización para la Cooperación y el Desarrollo Económicos (OCDE), ha fomentado la creación de PISA para el Desarrollo (PISA-D), iniciativa desarrollada para dar respuesta a la demanda de innovaciones que maximicen el acceso universal al aprendizaje de la lectura y las matemáticas. Todos los instrumentos y enfoques puestos a prueba en PISA-D, se incorporarán al estudio PISA, a partir del año 2021, OCDE (2017).

En el área de matemáticas, se enfatiza la importancia de utilizar una gran variedad de contextos apoyados por la tecnología, como una manera aceptada de entender el objetivo principal de adquirir aprendizaje, si bien se reconoce la importancia de resolver operaciones aritméticas, estas no son suficientes. Con la finalidad de aplicar estos conocimientos, es imprescindible tener capacidades básicas para elegir el modelo adecuado y seleccionar una estrategia o una explicación. Estas habilidades constituyen la esencia de lo que PISA, entiende por competencia matemática. El constructo de competencia matemática definido en PISA-D, destaca la necesidad de desarrollar la capacidad de los alumnos para utilizar las matemáticas en contexto enfatizando la realización de experiencias enriquecedoras en sus clases. En PISA-D la competencia matemática se define como se muestra en el figura 3 (OCDE, 2017).

Figura 3

Un modelo de competencia

matemática en la práctica

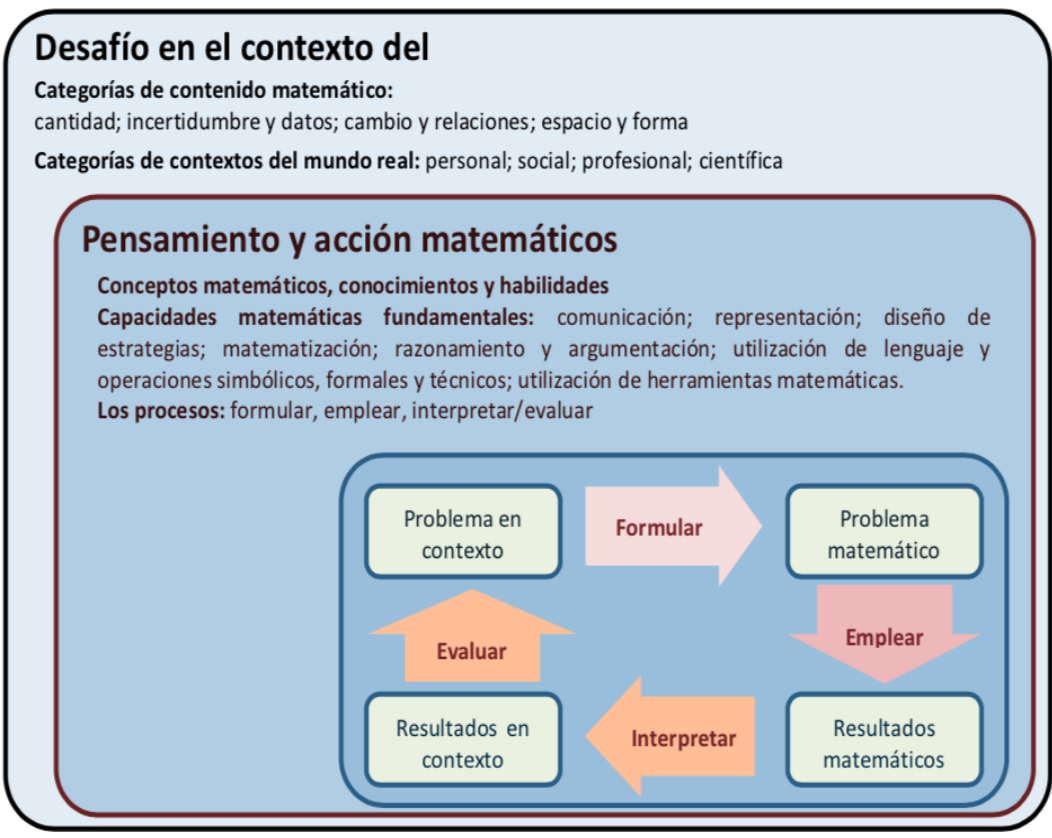

Fuente: OECD (2013).

\subsection{Perspectivas de futuro}

El alumno del siglo XXI, debe desarrollar nuevas competencias y habilidades, para encarar una transición cultural, caracterizada por una disrupción tecnológica, y por cambios paradigmáticos. Debe llegar a ser análitico, crítico, capaz de extraer conclusiones, a partir de la información obtenida del entorno que lo rodea, es importante que el estudiante encuentre la solución de un problema y, también, que, siempre que sea posible, busque varias formas de solución e investigue otras conexiones o extensiones del problema. Es en estas situaciones, cuando el uso de herramientas tecnológicas se transforma en un medio ideal para que el educando optimice sus esquemas, a través de sistemas de representación de los contenidos (Camacho y Santos, 2004; Alfaro at al., 2004; Gamboa 2007).

En la actualidad, la tecnología ha generado cambios en la forma cómo los estudiantes aprenden matemáticas. Los ambientes computacionales proporcionan condiciones para que los estudiantes identifiquen, examinen y 
comuniquen, distintas ideas matemáticas en un contexto cercano al real, la tecnología, puede llegar a ser una poderosa herramienta para que los estudiantes logren crear diferentes representaciones de ciertas tareas sirviendo como un medio para formular sus propias preguntas o problemas, lo que constituye un importante aspecto en el aprendizaje (Gamboa 2007; Barrera y Santos, 2001; Costa y Domingos 2019; Serhan 2019).

Pero debemos ser conscientes que, en la actualidad, existe una disponibilidad de una cantidad cada vez mayor de tecnologías sofisticadas, con avances relacionados en la medición, creación y entrega de evaluaciones automatizadas de matemáticas, que se basan en secuencias de elementos de prueba atomizados, que en muchas ocasiones se centran en la evaluación de objetivos de orden inferior, como la reproducción de conocimientos, habilidades y procedimientos basados en cálculos. Frente a este escenario los docentes se enfrentan al desafío del diseño de ejercicios contextualizados de resolución de problemas originales, no rutinarios y nuevos para los estudiantes (Hoogland, Tout 2018).

Es en este punto donde surge la interrogante, ¿cómo el profesor contextualiza los ejercicios matemáticos, a una realidad social y cultural cercana al alumno?, cada aprendiz posee vivencias y experiencias únicas. El docente deberá contextualizar ejercicios por cada alumno, por cada curso, por cada establecimiento de educación donde imparte clases. En la actualidad la tecnología ha influido en varios aspectos de la evaluación en la educación matemática: apoyando la medición de habilidades del pensamiento de orden superior, representando problemas auténticos del mundo que nos rodea para usar y aplicar conocimientos y habilidades matemáticas, entregando pruebas y análisis de resultados sofisticados en forma automática (Cóndor at al., 2020).

Este es un punto crítico de tensión, donde los ejercicios matemáticos contextualizados necesitan la tecnología para ser realizados, el docente propone un problema matemático, el sistema informático a través de parámetros ingresados con anterioridad (contexto social y cultural) adaptan el problema formulado por el docente a contextos cercanos al alumno, formulando pautas y guías de trabajo que apoyen al aprendiz en la formulación de una respuesta al problema planteado.

Lo expuesto no está lejano a la realidad en la actualidad, existen diferentes entidades desarrollando herramientas computacionales a nivel mundial:

La Universidad de Cataluña y el Departamento de Matemática de la Universidad Autónoma de Barcelona desarrollan AgentGeom, software que tiene como propósito ayudar a los alumnos en la apropiación de habilidades estratégicas y argumentativas implicadas en la resolución de problemas geométricos.

AgentGeom, contribuye de manera autónoma en las competencias que debe poseer un estudiante para resolver un problema, creando condiciones interactivas para que el alumno pueda analizar el proceso de solución, brinda apoyo al estudiante sólo con información limitada, con la finalidad de favorecer un tipo de aprendizaje constructivista. Genera, habilidades argumentativas en matemática, contando para ello con un área deductiva, donde el alumno dicta sentencias escritas siguiendo las normas del lenguaje matemático, desarrollando la capacidad de abstracción y las ideas relacionadas con la demostración en matemática.

El software AgentGeom simula la conducta de un tutor humano en tres aspectos:

- Autonomía en el sentido de mostrar cierta iniciativa y comportamientos calificables como espontáneos.

- Se desempeña como tutor; guía al estudiante y le proporciona mensajes de ayuda.

- Cumple la función de mediador al recibir las entradas de los alumnos y del profesor, validando las acciones del estudiante.

Este sistema experto, es una herramienta de apoyo al docente, con la finalidad de servir de soporte en el proceso de diversificación de contextos sociales y culturales de los alumnos en el aula. Este tipo de herramienta puede 
contribuir decisivamente con el desarrollo de las habilidades del pensamiento y destrezas cognitivas, que fortalecen la capacidad de razonamiento, la disciplina mental y el rigor en la toma de decisiones.

El Instituto Alemán de Investigación de Inteligencia Artificial (DFKI), ha desarrollado una aplicación educativa para las matemáticas, Active Math y MathBridge, innovador sistema de tutoría inteligente, basado en la web, el cual incluye técnicas de inteligencia artificial, como adaptabilidad al contexto del usuario, generación de cursos, modelado de estudiantes, herramientas cognitivas, generación de ejercicios, generación de retroalimentación, diagnóstico y estrategias de tutoría.

Este sistema genera cursos que proporcionan adaptabilidad al contexto del alumno, es decir, estos cursos dependen de los objetivos del aprendiz, su nivel de dominio de la materia y preferencias preestablecidas. De esta manera, el ritmo de aprendizaje, el nivel de detalle, la forma de presentar el contenido, el formato de presentación y el número y la dificultad de los ejemplos y ejercicios se pueden personalizar para el alumno individual según sea necesario. ActiveMath puede trabajar con varios sistemas de álgebra computacional, usa y actualiza un modelo de aprendizaje simple. Proporciona un diccionario, notas públicas y privadas, anotaciones adaptativas de tablas de contenido, etc. La adaptación a las capacidades del alumno se produce tanto en la generación del curso como en el mecanismo de sugerencia. El curso comprueba, si el nivel de dominio de los conceptos de prerrequisito es suficiente para la competencia objetivo. En caso contrario, presenta los conceptos y / o explicaciones que faltan, ejemplos y ejercicios para estos conceptos al alumno cuando se solicita una nueva sesión. El mecanismo de sugerencia actúa dinámicamente en respuesta a las actividades del estudiante. Básicamente, este mecanismo funciona con dos pizarrones, un pizarrón de diagnóstico y un pizarrón de sugerencias sobre el que operan determinadas fuentes de conocimiento.

Las investigaciones sobre pedagogía en las matemáticas, han reconocido que los estudiantes, aprenden de manera más efectiva, si el aprendizaje tradicional de memoria de fórmulas y procedimientos se complementa con la posibilidad de explorar una amplia gama de problemas y situaciones reales. La enseñanza, con una orientación hacia la resolución activa de problemas, produce mejores resultados de aprendizaje en el sentido de que el conocimiento adquirido está más disponible y es aplicable especialmente en contextos conocidos, ya que una reflexión sobre las actividades y métodos de resolución de problemas produce una comprensión más profunda y un mejor desempeño (Er y Siekmann, 2004). Los alumnos crecen y aprenden en una red compleja e interconectada de relaciones, experiencias, contextos sociales y culturales, en 1977, Urie Bronfenbrenner, presentó un argumento innovador y poderoso: una comprensión completa del desarrollo humano, requiere que vayamos más allá de las simples relaciones uno a uno, entre los alumnos y su entorno inmediato, proponiendo que examinemos los entornos complejos e interrelacionados en los que viven y los contextos más amplios que pueden afectarlos. La teoría de los sistemas Bio-ecológicos que se desarrolló a partir de esta propuesta se ha convertido en una importante herramienta influyendo como base teórica en investigaciones científicas (Shonkoff y Phillips, 2000).

En el área de matemáticas, los alumnos se enfrentan a la resolución de problemas abstractos, pero en el mundo real los alumnos deben resolver problemas influenciados por sus entornos domésticos y escolares, las políticas y prácticas que conforman estos entornos, los valores culturales que los sustentan y las complejas relaciones entre estos factores. Por ello, los autores de este artículo plantean un modelo teórico para la resolución de problemas matemáticos en contextos apoyados por sistemas tecnológicos de tutoría inteligente (ITS) basado en el modelo Bio-ecológico de Sistemas Ambiente de Bronfenbrenner. (Figura 4) 
Figura 4

Modelo teórico para la resolución de

problemas matemáticos en contextos

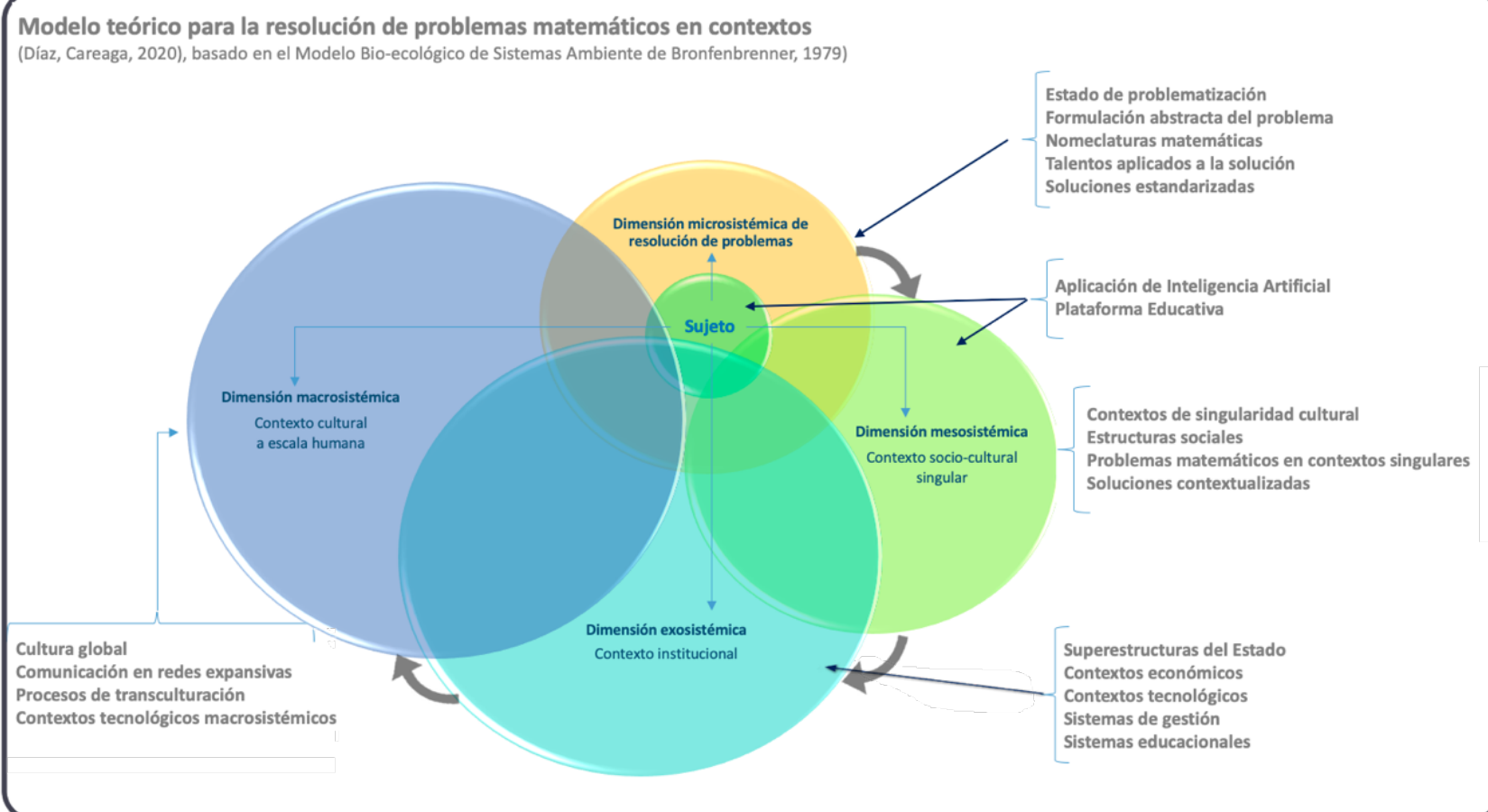

Fuente: Elaboración propia (2020) basada en el Modelo Bio-ecológico de Bronfenbrenner, 1979

- El microsistema, es el primer círculo que rodea al alumno, donde se encuentran el estado de problematización como una formulación abstracta del problema, los problemas se autodeterminan por sus nomenclaturas matemáticas; los talentos de los sujetos son aplicados a la resolución, a través de soluciones estandarizadas, lo cual tiende a dejar los problemas matemáticos desafectos de su aplicabilidad en contextos.

Los factores intervinientes, para relacionar los problemas matemáticos abstractos con los contextos, pueden ser mediatizados por soluciones tecnológicas basadas en Inteligencia Artificial y el uso de Plataformas Educativas.

- El siguiente círculo mesosistema, reconoce las relaciones entre los entornos del microsistema, y las condiciones externas del mesosistema. Estos contextos, que influyen en la aplicabilidad de los problemas matemáticos, se caracterizan por estar remitidos a la singularidad cultural del grupo humano al que pertenecen los sujetos y las estructuras sociales. Mediatizados los problemas matemáticos con IA y Plataformas Educativas se provocan interrelaciones entre los problemas, sus distintas alternativas de solución y las determinantes emanadas de las singularidades, lo que posibilitará soluciones contextualizadas.

- El exosistema, se enfoca en contextos amplios, se refiere a las fuerzas que son influyentes en sucesos en los microsistemas, el individuo no es entendido como un sujeto activo, lo conforma la naturaleza del trabajo de los progenitores, en el ámbito de la educación, se expresa a través de las relaciones que mantiene un profesor con el resto del claustro, con el apoyo de la ejercitación de problemas matemáticos contextualizadas con IA y Plataformas Educativas.

- Finalmente, el círculo, llamado macrosistema, lo configuran la cultura y la subcultura en la que se desenvuelven las personas y todos los individuos de la sociedad. Las condiciones sociales y estructurales que determinan los rasgos generales de las instituciones y contextos, en donde se desarrolla el sujeto. 
Lo constituye los valores propios de una cultura, costumbres, ideología, valores, leyes, regulaciones y las costumbres de una cultura particular.

A través de este modelo teórico los ITS deben considerar estos cuatro estados en el diseño lógico antes de la implementación física y aplicación del software de resolución de problemas matemáticos en contexto.

Con el desarrollo, y la creciente disponibilidad de programas tutores, la aplicación de sistemas de aprendizaje inteligentes se vuelve más probable y precisa, la investigación de características inteligentes recibe más atención que en el pasado. Como resultado, se han establecido una serie de nuevos sistemas de tutoría inteligente con entornos de aprendizaje adaptativo para el alumno. Estos sistemas se esfuerzan por mejorar la enseñanza a distancia, por complementar la enseñanza tradicional en el aula y por apoyar el aprendizaje individual, permitiendo al usuario, aprender en su propio entorno social y cultural (Naser, 2016). La finalidad, de los sistemas de tutoría inteligente es guiar a los estudiantes en el proceso de ejercitación y comprensión de las matemáticas, basado en la experiencia previa de los alumnos (Chassignol at al., 2018).

\section{Conclusiones}

Se debe destacar, que es necesario desarrollar conciencia entre académicos y estudiantes, respecto a la resolución de problemas matemáticos, en contexto y su papel en el ámbito de la educación, entendiendo que ellas deben apuntar a la mejora de las prácticas implementadas en el aula. La innovación en el desarrollo de problemas matemáticos es un proceso que debe ser fruto de una profunda reflexión, en donde se tengan en cuenta variables humanas y educativas que validen su realización.

Se debe reconocer que la utilización de problemas en contexto, es un proceso de aplicación de conocimientos previamente adquiridos a situaciones nuevas y desconocidas donde el objetivo principal de la enseñanza matemática, es que los estudiantes deben convertirse en solucionadores de problemas. Sin embargo, dadas las múltiples interpretaciones del término, el objetivo no está claro, la enseñanza matemática depende de la conceptualización de lo que es la matemática y de lo que significa comprender la resolución de problemas. Para algunos el conocimiento en esta área es visto como un conjunto de hechos y procedimientos que tratan con cantidades, magnitudes y formas, y las relaciones entre ellas; para otros, las matemáticas se conceptualizan como la ciencia de los patrones, una disciplina empírica que pone su énfasis en la búsqueda de reglas sobre la base de la evidencia empírica.

El papel de la tecnología en esta área, es el apoyo al aprendizaje matemático, mejorar el pensamiento y aumentar el proceso educativo, se tiende a confundir o dar mal uso a la tecnología, al utilizarla como un conjunto de procedimientos para la entrega, control y evaluación de contenido. El uso de la tecnología, en educación y su relación con la innovación de las prácticas pedagógicas agregan otra complejidad, ya que no siempre se logra establecer una sintonía entre el uso de TIC y la innovación educacional. Los cambios que se están produciendo en la sociedad inciden en la demanda de una redefinición del trabajo del profesor y de la profesión docente, de su formación y de su desarrollo profesional (Careaga y Avendaño, 2017). Es en este punto donde la inteligencia artificial en la educación (AIED) se presenta como un gran apoyo al docente en el proceso de redefinición, ofreciendo experiencias de aprendizaje más flexibles e inclusivas, personalizadas, efectivas y atractivas en entornos formales e informales desarrollando sistemas de tutoría más dinámicos y adaptativos al alumno.

La IAED como apoyo al proceso de contextualización en la resolución de problemas matemáticos, abren nuevas posibilidades en el proceso de ejercitación y evaluación de habilidades del pensamiento de orden superior en matemáticas, representando problemas auténticos del mundo que nos rodea para usar y aplicar conocimiento y habilidades matemáticas, generando una entrega de resultados personalizados y oportunos. 


\section{Referencias bibliográficas}

Alfaro, A., Alpízar, M., Arroyo J., Gamboa R., Hidalgo M. (2004). Enseñanza de las Matemáticas en Costa Rica: Elementos para un Diagnóstico. Universidad Nacional, Heredia, Costa Rica.

Aravena, M. y Caamaño, E. (2007). Modelización matemática con estudiantes de secundaria de la comuna de Talca, Estudios Pedagógicos XXXIII, No 2: 7-25.

Arcavi, A. y Friedlander, A. (2007). Curriculum developers and problem solving: the case of Israeli elementary school projects. ZDM The International Journal on Mathematics Education, 39, 5-6, pp. 355-364.

Barrera, F. y Santos, M. (2001). Students use and understanding of different mathematical representations of tasks in problem solving instruction. Proceedings of the Twenty-Three Annual Meeting North American Chapter of the International Group for the Psychology of Mathematics Education. Vol. 1, pp. 459-466. ERIC Clearinghouse for Science, Mathematics, and Environmental Education.

Biembengut, M. (2007). Modelling and applications in primary education. In W. Blum, P. L. Galbraith, H.-W. Henn, y M. Niss (Eds.), Modelling and applications in mathematics education (pp. 451-456). New York: Springer.

Boaler, J. (1993). Encouraging the transfer of 'school' mathematics to the 'real world' through the integration of process and content, context and culture. Educ Stud Math 25, 341-373. https://doi.org/10.1007/BF01273906

Bronfenbrenner, U. (1979). La ecología del desarrollo humano. Barcelona: Editorial Paidós.

Chassignol, M., Khoroshavin, A., Klimova, A., Bilyatdinova, A. (2018). Artificial Intelligence trends in education: a narrative overview, Computer Science, Volume 136, Pages 16-24, ISSN 1877-0509, https://doi.org/10.1016/j.procs.2018.08.233.

Callejo, L. (1998). Un club matemático para la diversidad. Madrid: Narcea.

Camacho, M. y Santos, M. (2004). El estudio de fenómenos de variación haciendo uso de herramientas tecnológicas. Uno Revista Didáctica de las matemáticas. No 37, pp. 105-122.

Careaga, M. y Avendaño, V. (2017). Currículum Cibernético y Gestión del Conocimiento. Fundamentos y Modelos de Referencia. Chile: RIL Editores y Editorial UCSC. ISBN: 978-956-01-0397-0

Castaño, N. (2014). Dificultades en la enseñanza de las operaciones con números racionales en la educación secundaria. Recuperado de: http:// repositorio.autonoma.edu.co/jspui/bitstream/11182/861/1/Tesis\%20 N\%C3\%A9stor\%20Mario\%20Casta\%C3\%B1o.pdf

Cóndor, O., Jadán J., Ramos C. (2020) Objetos virtuales de aprendizaje del proceso educativo matemático. En: Karwowski W., Ahram T., Etinger D., Tanković N., Taiar R. (eds) Ingeniería y diseño de sistemas humanos III. Avances en Computación y Sistemas Inteligentes, vol 1269. Springer, Cham.

https://doi.org/10.1007/978-3-030-58282-1_31

Costa, M. y Domingos A. (2019). Technology as a resource to promote mathematics Teaching. Universidade Nova de Lisboa. Recuperado de: http://hdl.handle.net/10362/96305

Crespo, S. (2003). Learning to pose mathematical problems: exploring changes in preservice teachers practises, Educational Studies in Mathematics, vol. 52, pp. 243-270.

D’Amore, B. y Pinilla, F. (2001). Matemática de la cotidianidad. Paradigma. Maracay, Venezuela. XXII, 1, 59-72.

Darragh, L. (2016). Identity research in mathematics education. Springer Science Business Media Dordrecht. 
De Lange, J. (1987). Language and Mathematics: Bridging between Natural Language and Mathematical Language in Solving Problems in Mathematics. Mathematics insight and meaning. Utrect, Holland: Rijksuniversiteit.

Delgado, J. (1999). La enseñanza de la Resolución de Problemas Matemáticos. Dos elementos fundamentales para lograr su eficacia: La estructuración del conocimiento y el desarrollo de habilidades Generales matemáticas. Tesis Ph. D. ISPJAE. Ciudad Habana. Cuba.

Díaz, C. (2003). Heurísticas y sesgos en el razonamiento probabilístico. Implicaciones para la enseñanza de la Estadística, en Actas del 27 Congreso Nacional de Estadística e Investigación Operativa, pp. 3611-3621.

Díaz, C. (2018). Investigación cualitativa y análisis de contenido temático. Orientación intelectual de revista Universum. Revista General De Información Y Documentación, 28(1), 119-142. https://doi.org/10.5209/RGID.60813

Díaz, M. y Poblete, A. (2001). Contextualizando tipos de problemas matemáticos en el aula. Revista de didáctica de las matemáticas, ISSN 0212-3096, ISSN-e 1887-1984, №. 45, 2001, págs. 33-42.

Díez, J. (2004), L'ensenyament de les matemàtiques en l'educació de persones adultes. Un model dialògic, tesis doctoral, Barcelona, Universitat de Barcelona.

Er, Melis y Siekmann, Jörg. (2004). ACTIVEMATH: An intelligent tutoring system for mathematics. LNCS (LNAI). 91-101. 10.1007/978-3-540-24844-6_12.

Font, V. (2007). Comprensión y contexto: una mirada desde la didáctica de las Matemáticas. La Gaceta de la Real Sociedad Matemática. Española, vol. 10, núm. 2, pp. 419-434.

Fuenlabrada, I. (2005). Aprender a enseñar matemáticas. Monterrey: Centro de altos estudios e investigación pedagógica.

Gamboa, R. (2007). Uso de la tecnología en la enseñanza de las matemáticas. Cuadernos de investigación y formación en educación matemática, 2 (3), 11-44.

Graumann, G. (2011). Mathematics for problem in the everyday world. In J. Maasz y J. O'Donoghue (Eds.), Realworld problems for secondary school mathematics students: case studies (pp. 113-122). Rotterdam: Sense Publishers.

Gravemeijer, K. y Doorman, M. (1999). Context problems in realistic mathematics education: A calculus course as an example. Educational Studies in Mathematics, 39(1-3), pp.110-128.

Greer, B., Verschaffel L., y Mukhopadhyay, S. (2007). Modelling for life: Mathematics and children's experience. In W. Blum, P. L. Galbraith, H.-W. Henn y M. Niss (Eds.), Modelling and applications in mathematics education (pp. 89-98). New York: Springer.

Hoogland, K., Tout D. (2018). Computer-based assessment of mathematics into the twenty-first century: pressures and tensions. ZDM Mathematics Education 50, 675-686 https://doi.org/10.1007/s11858-0180944-2

Informe Cockcroft, W. H. (1985). Las matemáticas sí cuentan. Madrid: MEC. Recuperado de: https://redined.mecd.gob.es/xmlui/handle/11162/105644

Jaworski, B. (2006). Theory and Practice in Mathematics Teaching Development: Critical Inquiry as a Mode of Learning in Teaching. Journal of Mathematics Teacher Education 9, 187-211 (2006). https://doi.org/10.1007/s10857-005-1223-z 
Jones, G., Langrall, W. y Money, E. (2007). Research in probability. Responding to classroom realities, en F. K. Lester (ed.), Second Handbook of Research on Mathematics Teaching and Learning, Charlotte, Information Age Publishing.

Kurt G. (1931), On formally undecidable propositions of Principia Mathematica and related systems I, Mathematical Logic, 1879-1931, Harvard University Press, Cambridge, pp. 596-616.

Lerman, S. (2001). Cultural, discursive psychology: a sociocultural approach to studying the teaching and learning of mathematics. Educational Studies in Mathematics 46(1-3) 87-113.

Lesh, R. y Zawojewski, J. (2007). Problem Solving and Modelling, in F. Lester, ed. The Handbook of Research on Mathematics Teaching and Learning. Reston, Virginia. Publishing, pp. 763-804.

Leal, H. y Bong S. (2015). The mathematical problem solving in the context of learning projects. Revista de Investigación, 39(84), 71-93.

Lithner, J. (2004). Mathematical reasoning in calculus textbook exercises, Journal of Mathematical Behavior, vol. 23, pp. 405-427.

Liljedah, P., Santos, M., Malaspina, U., y Bruder, R. (2016). Problem Solving in Mathematics Education. Hamburg: Springer Open.

Maass, K. (2010). Classification scheme for modelling tasks. Journal für Mathematik-Didaktik, 31(2), 285-311.

MINEDUC (2019). Organización Curricular Matemáticas. Recuperado de:

https://www.curriculumnacional.cl/portal/Educacion-General/Matematica/20852:Organizacion-CurricularMatematicas

Mthethwa, T. (2019). Mathematical literacy, mathematics in context: whose context is this for?. Proceedings of the 25th Annual National Congress of the Association for Mathematics Education of South Africa Developing Deep Mathematical Thinking through Mathematics Teaching. University of KwaZulu-Natal.

Naser, S. (2016). ITSB: An Intelligent Tutoring System Authoring Tool. Journal of Scientific and Engineering Research. 3(5):63-71. ISSN: 2394-2630 Recuperado de: https://core.ac.uk/download/pdf/131208699.pdf

OECD (2013), PISA 2012 Assessment and Analytical Framework: Mathematics, Reading, Science, Problem Solving and Financial Literacy, p.26, OECD Publishing. Recuperado de:

http://dx.doi.org/10.1787/9789264190511-en

OECD (2014). Skills for Life: Student Performance in Problem Solving. PISA 2012 Results, vol. VI. Paris: OECD. Recuperado de: http://www.oecd.org/pisa/keyfindings/pisa-2012-results.htm

OCDE (2017), Marco de Evaluación y de Análisis de PISA para el Desarrollo : Lectura, matemáticas y ciencias, Versión preliminar, OECD Publishing, Paris.

Ortiz, P. (2005). Dificultades de aprendizaje e intervención psicopedagógica en la resolución de problemas matemáticos. Revista educación, 257-286.

Orton, A. (1998): Didáctica de las matemáticas. Ed. II España: Ediciones Morata, S.L. ISBN: 978-84-7112-604-7

Piñuel, J. (2002). Epistemología, metodología y técnicas del análisis de contenido. Estudios de Sociolingüística, $3(1), 1-42$.

Piaget J. (1969) Psicología y Pedagogía. Barcelona: Ariel. ISSN: 2477-8818 
PISA (2006). Programa para la Evaluación Internacional de Alumnos de la OECD. Recuperado de:

https://www.educacionyfp.gob.es/inee/dam/jcr:699d43f6-ddcc-4c7e-b7bf-

c0e0c288e949/pisainforme2006.pdf

PISA (2012). Resolución de problemas de la vida real. Resultados de matemáticas y lectura por ordenador. Informe español. Recuperado de:

https://sede.educacion.gob.es/publiventa/descarga.action?f_codigo_agc=16427

Pozo, J. y Monereo, C. (Ed.) (1999). El aprendizaje estratégico. Madrid, Aula XXI- Santillana.

https://dialnet.unirioja.es/servlet/libro?codigo=10213

Ramos, A. y Font, V. (2006). Contexto y contextualización en la enseñanza y el aprendizaje de las matemáticas. Una perspectiva ontosemiótica. Matemáticas y su didáctica, Año 20, N. 4, 535-556.

Russell B. (1903) The Principles of Mathematics. Trinity College, Cambridge.

Santos, M. (2007). Mathematical problem solving: an evolving research and practice domain. ZDM The International Journal on Mathematics Education, 39, 5- 6, pp.523-536.

Santos, M. (2008). La resolución de problemas matemáticos avances y perspectivas en la construcción de una agenda de investigación y práctica. Investigación en educación matemática XII, 2008, ISBN 978-84-9344889-9.

Schoenfeld, A. (1988). When good teaching leads to bad results: the disasters of "well taught" mathematics classes. Educational psychologist, 23(2), 145-166.

Serhan, D. (2019).Web-Based Homework Systems: Students' Perceptions of Course Interaction and Learning in Mathematics. International Journal on Social and Education. State University, USA Sciences. ISSN: 26887061

Shonkoff, J. y Phillips, D. (2000). From neurons to neighborhoods: The science of early childhood development. Washington, DC: National Academies Press

Silver, E. (1985). Teaching and learning mathematical problem solving: Múltiple research perspectives. Hillsdale, NJ: LEA.

Stein, M. K., Engle, R. A., Smith, M. S., \& Hughes, E. K. (2008). Orchestrating Productive Mathematical Discussions: Five Practices for Helping Teachers Move Beyond Show and Tell. Mathematical Thinking and Learning, 10(4), 313-340. https://doi.org/10.1080/10986060802229675

Van den Heuvel-Panhuizen M. (2001). Children learn mathematics. Utrecht/Enschede: Freudenthal Institute, Utrecht University/SLO.

Van den Heuvel-Panhuizen M. y Bodin-Baarends C. (2004). All or nothing: Problem solving by high achievers in mathematics. Journal of the Korea Society of Mathematical Education, 8(3), 115-121.

Vygotsky (1934/2009). El Desarrollo de los Procesos Psicológicos Superiores. Biblioteca de bolsillo, tercera edición, Editorial Crítica, Harvard University Press Cambridge, Massachusetts, traducción al español Silvia Furió. https://saberespsi.files.wordpress.com/2016/09/vygostki-el-desarrollo-de-los-procesospsicolc3b3gicos-superiores.pdf

Esta obra está bajo una Licencia Creative Commons Attribución-NoCommercial 4.0 International

(cc) BY-NC 